\title{
Stereo Vision Calibration Procedure for 3D Surface measurements
}

\author{
João L. Vilaça \\ Industrial Electronics Dept. \\ Minho University, Guimarães, \\ 4800-058 PORTUGAL \\ joaovilaca@dei.uminho.pt
}

\author{
Jaime Fonseca \\ Industrial Electronics Dept. \\ Minho University, Guimarães, \\ 4800-058 PORTUGAL \\ jaime@dei.uminho.pt
}

\author{
A. C. Pinho \\ Mechanical Engineering Dept. \\ Minho University, Guimarães, \\ 4800-058 PORTUGAL \\ acmpinho@dem.uminho.pt
}

\begin{abstract}
In reverse engineering, rapid prototyping or quality control with complex 3D object surfaces, there is often the need to scan a complete 3D model using laser digitizers. Those systems usually use one camera and one laser,using triangulation techniques; complex 3D objects can cause information gaps in the model obtained. To overcome this problem, another camera can be used. Traditional calibration procedures for those systems normally result in a full 3D camera calibration, involving individual implicit camera parameters, a rigid motion equation relating the explicit cameras parameters of each camera and the intersection with the laser line. This paper presents a new stereo vision laser strip calibration procedure for 3D surface measurements. In this calibration procedure the laser plane is the one that matters, only one set of laser-coplanar calibration points for camera calibration is needed, and a dead zone scan area is considered, since the digitalization arm is mounted in a 3 degree freedom machine PC-based Motion Control with multiple scan paths.
\end{abstract}

\section{INTRODUCTION}

Nowadays, reverse engineering from physical models is appealed with two principals and distinct objectives. The first one is the dimensional verification of the sculptured model, as way, to evaluate if eventual dimensional conditions are respected; the other one has as objective the creation of virtual models (CAD) once that, the intended form is geometric complex [1].

Some of these measurement techniques are based on scanning a surface, being characterized by a direct controlled projection of energy on the surface, with recourse to one or more projectors and one or more sensors. These techniques require complicated calibration procedures to evaluate the parameters of the optical devices (sensors and light source), necessary for the measurement process [2][3].

Camera calibration is understood as the process of determining the internal camera geometric and optical characteristics (intrinsic parameters) and/or 3D position and orientation of the camera frame relative to a certain world coordinate system (extrinsic parameters). The propose of the calibration it to establish the relationship between 3D world coordinates and their corresponding 2D image coordinates as seen by the computer [5][6][7].

In systems that use a laser light and a camera for 3D measurements, the common procedure is to establish a relationship between 2D images coordinates and a 3D world coordinates using a triangulation procedure [8].

For 3D measurement systems that use stereo vision, the matching of conjugate image points is required. The correlation process is typically based on similarity constrains (feature-based or intensity-based). However in epipolar geometry, a point in an image from camera 1 can be correlated with many points in the image from camera 2 (false matches), and this problem is particularly evident in measuring free-form surfaces due to the absence of peculiarities in the scene to be used as similarity constrains [4]. Some calibration procedures resolve the matching point problem intersecting the epipolar line with the laser line in the image [3].

This paper presents a research activity concerning the development of a simple and versatile stereo vision calibration procedure for non-contact laser scanning system. It is organized in the following sections: stereo calibration procedure, divided in three steps: horizontal image correction, vertical image correction and real world coordinates correspondence, all these steps are based on polynomial correction; laser stripe detection; results for the system's accuracy and a conclusion.

\section{CALIBRATION PROCEDURE}

Most of the techniques used for camera calibrations processes are normally based on the pinhole camera model. They define the calibration parameters $\mathrm{R}$ (rotation matrix) and $\mathrm{T}$ (translation vector), allowing, through a rigid equation, the transformation of the object world coordinate system to the camera 3D coordinate system. After this, they transform the 3D camera coordinate to an ideal (undistorted) image coordinate using perspective projection with pinhole camera geometry, calibrating the intrinsic camera parameters. They include modeling for lens distortion, which can be of two kinds: radial and tangential. 
In the calibration procedure presented here only radial distortion will be considered, because it is that which needs to be considered in most of the industrial machine vision applications, and only for the laser plane.

The system is constituted by two CCD FIREWIRE cameras, with resolution $640 \times 480$, using $8 \mathrm{~mm}$ lenses and a laser with $1 \mathrm{~mW}$ power. The laser is located at the same physical distance from both cameras, and the cameras are guided at $30^{\circ}$ to the horizontal line (Fig. 1). Although mechanically the system is presented as calibrated (distances cameras-laser, camera angles and laser line parallel to cameras), the comparison of results from the images of both cameras showed that in the laser plane (plane studied for calibration) the system was far from calibrated, presenting the following problems:

- A point in the $\mathrm{O}_{\text {laser }}$ coordinated system corresponded to a point in the $\mathrm{O}_{1}$ coordinated system and other point in the $\mathrm{O}_{2}$ coordinated system, being the $\mathrm{O}_{1}$ point different from the $\mathrm{O}_{2}$ point.

- Perfectly visible radial distortion in some parts of the image, adding to the previous problem.

- Creation of image dead zones - problem caused by the relative angle of the cameras to the laser plane.
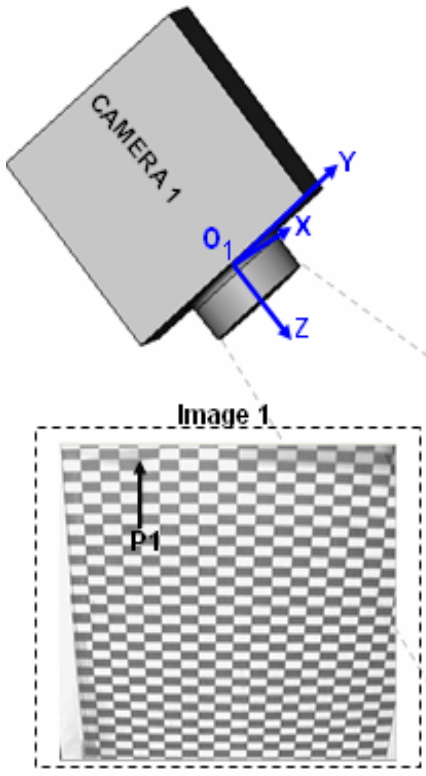

Image 1 - calibrated

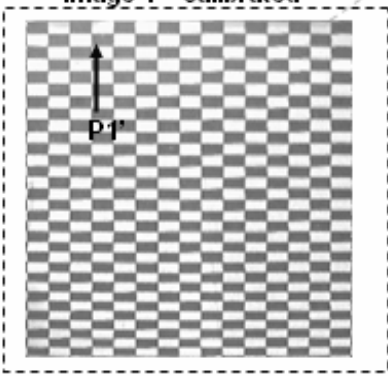

P1'(image1 - calibrated) = P2' (image2 - calibrated
This angle means that in the upper part of the image the horizontal distance was less than the horizontal distance in the lower part of the image, relative to $\mathrm{O}_{\text {laser }}$ coordinated system. This problem only matters because the system to be calibrated will be assembled in a $3 \mathrm{D}$ freedom machine that will carry out readings on parallel surface planes.

To avoid these problems a calibration procedure was developed that calibrates each one of the images, verifying that a point in $\mathrm{O}_{1}$ is equal to a point in $\mathrm{O}_{2}$, eliminating the radial distortion on the lenses and image dead zones.

For calibration a pattern was created with $10 \times 5 \mathrm{~mm}$ rectangles. The pattern was printed on an acetate sheet and placed between two glass plates with thickness of $1.2 \mathrm{~mm}$, all the rectangles being co-planar and visible to both the cameras. The pattern was placed in the laser plane (Fig. 1) making it easy to visualize the correspondence between the images points of both cameras, which is the basis of this calibration procedure.

The calibration procedure is divided into three steps for both images: horizontal image correction (A), vertical image correction (B) and real world coordinates correspondence $(\mathrm{C})$.

Fig.1 - Physical disposition of the calibration process. Correspondence of one point in $\mathrm{O}_{\text {laser }}$ plane in the two camera images before and after calibration. 


\section{A. Horizontal image correction}

The horizontal image correction (Fig. 2) for each camera is divided into five steps, in which the collected points in the image of each camera will have to correspond to the same points in the laser plane:

1. Acquisition of a set of vertical image points which will be the horizontal zeros of the image after the horizontal correction.

2. Polynomial determination that translates the relation between the position (y) of vertical points and the position (x) of horizontal points (1).

3. Acquisition of a set of vertical image points, which will be the horizontal limits of the image after horizontal correction.

4. Polynomial Determination that translates the relation between the position (y) of vertical points and the position (x) of horizontal points (2).

5. Creation of a new horizontally corrected image, with horizontal dimension given by (3), where the position in $\mathrm{x}$ of each image pixel, relatively to the not horizontally corrected image is given by (4), maintaining equal the related pixel position in $y$.

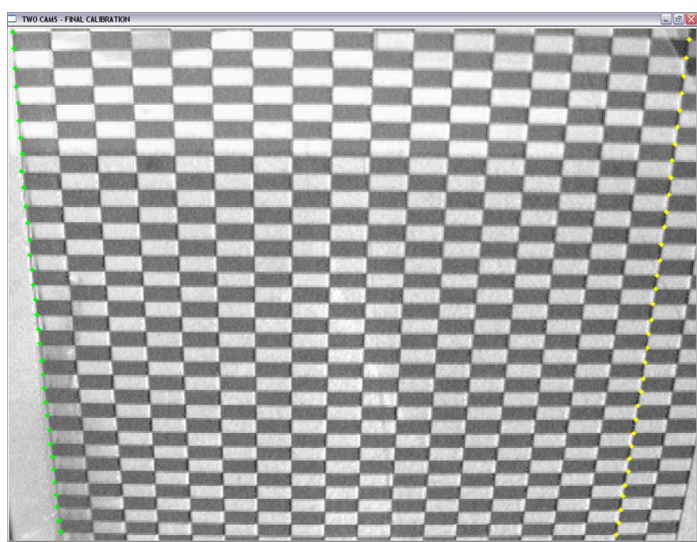

Fig. 2 - Vertical point extraction from image for horizontal image correction.

$$
\begin{aligned}
& x_{\text {initial }, \text { cam }}(y)=f(y) \\
& x_{\text {final,cam }}(y)=f(y) \\
& I_{\text {Widthc }}=W_{\text {max rectan gle }} \times N_{\text {rectan gles }} \\
& x_{c, \text { cam }}(x, y)=\frac{x-x_{\text {initial }}(y)}{I_{\text {Widthc }} / x_{\text {final }}(y)-x_{\text {initial }}(y)} \\
& 0 \leq x<I_{\text {Width }} ; 0 \leq y<I_{\text {Height }} ; 1 \leq \text { cam } \leq 2
\end{aligned}
$$

\section{B. Vertical image correction}

Vertical image correction (Fig. 2) from the horizontally corrected image is divided into five steps, in which the points compiled in the horizontally corrected image will have to correspond to the same points in the laser plane:

1. Acquisition of a set of horizontal image points which will be the vertical zeros of the image after vertical correction.

2. Polynomial determination that translates the relation between the position (x) of the horizontal points and the position (y) of the vertical points (5).

3. Acquisition of a set of horizontal image points, points these, which will be the vertical limits of the image after vertical correction.

4. Polynomial determination that translates the relation between the position $(\mathrm{x})$ of horizontal points and the position (y) of vertical points (6).

5. Creation of a new vertically corrected image, with horizontal dimension given by (7), where the position in $\mathrm{y}$ of each image pixel relative to the horizontally corrected image is given by (8), maintaining equal the related pixel position in $\mathrm{x}$.

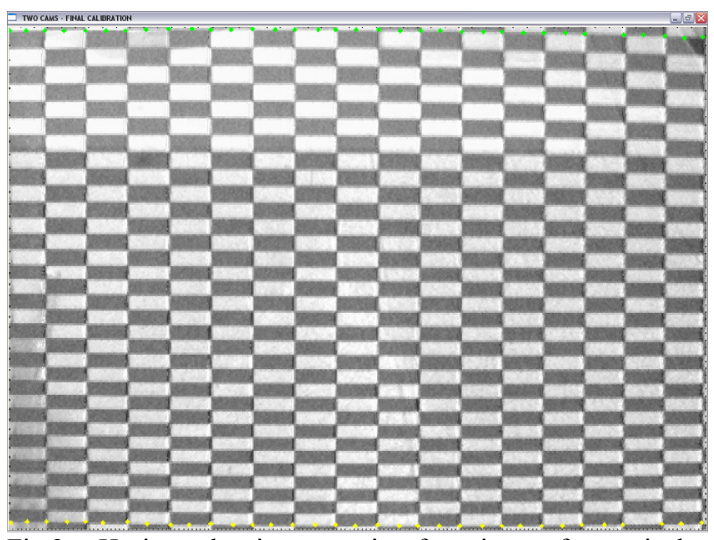

Fig. 3 - Horizontal point extraction from image for vertical image correction.

$$
\begin{aligned}
& y_{\text {initial }, \text { cam }}(x)=f(x) \\
& y_{\text {final }, \text { cam }}(x)=f(x) \\
& I_{\text {Heightc }}=H_{\text {max rectan gle }} \times N_{\text {rectan gles }}(7) \\
& y_{\text {c,cam }}(x, y)=\frac{y-y_{\text {initial }}(x)}{I_{\text {Heightc }} / y_{\text {final }}(x)-y_{\text {initial }}(x)} \\
& 0 \leq x<I_{\text {Widthc }} ; 0 \leq y<I_{\text {Height }} ; 1 \leq \text { cam } \leq 2
\end{aligned}
$$


Polynomial determination uses the method of the minimum squares to calculate the adjustment through points, using (9).

$$
y=b+c_{1} x+c_{2} x^{2}+c_{3} x^{3}
$$

$b$ and $c_{1} \ldots c_{3}$ are constant

The point's selection for horizontal and vertical image correction is carried through manually, being that, the pattern selected points in the image 1 have to correspond to the same pattern selected points in the image 2 .

\section{Real world coordinates correspondence}

The correspondence between the corrected image points and the points of $\mathrm{O}_{\text {Laser }}$ plane, in $\mathrm{x}$ coordinate, is given by (10), where $\mathrm{k}$ is a constant determined by the difference in distance between the two points in the corrected image and the distance between the two points in the pattern, points that will have to correspond to one of the rectangle vertices in the same horizontal edge of the pattern.

$$
x_{\operatorname{Re} a l}=k \times x, \quad 0 \leq x<x_{\text {Widthc }}
$$

In y coordinate, the correspondence is given by (11), where $\mathrm{f}(\mathrm{y})$ is a polynomial. This polynomial is defined by the acquisition of a set of vertical points in the corrected image, points that will have to correspond to the different horizontal pattern edges visualized in the corrected image, making possible its correspondence with real values, given that the real distance between edges is known.

$$
y_{\operatorname{Re} a l}(y)=f(y), \quad 0 \leq y<Y_{\text {Heightc }}
$$

\section{LASER STRIPE DETECTION}

The laser line detection is executed in two steps.

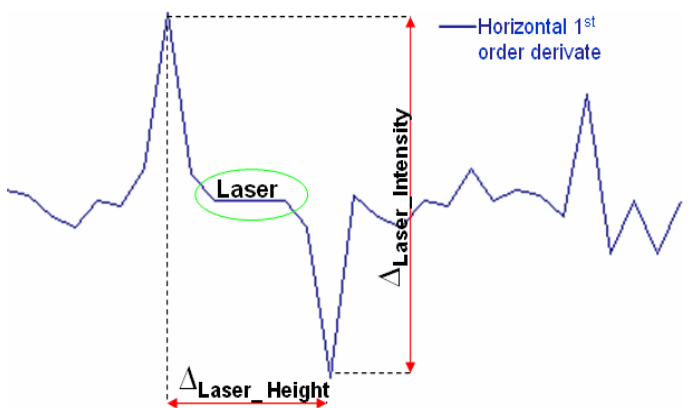

Fig.4 - Representation of image horizontal first order derivate and laser line detection.

The first step is taken before the calibration process, and for each one of the camera images.
It is characterized by the image horizontal first derivative calculation. Thus, each image column is derived (Fig. 4) and detected; the laser using three validation conditions:

1. Detection in the signal of positive for negative type transitions, and detection of the respective peaks;

2. To verify if the distance between peaks is greater than $\Delta_{\text {Lazer_Height }}$ - maximum number of horizontal laser pixels;

3. Calculating the amplitude between peaks and verification if the amplitude is greater than $\Delta_{\text {Laser_Intensity }}$ - laser minimum intensity relative to the surface on which it occurs.

If the three conditions are validated, the vertical pixels of the analyzed column between transitions are placed in white and all the remainder in black. In the event of detecting more than one zone with validated parameters in the same column, the one with greater $\Delta_{\text {Laser_Intensity }}$ is chosen. The value of the parameter $\Delta_{\text {Lazer Height }}$ is attributed in function of the vertical resolution of the camera, the laser power and the distance camera/laser. The value of the parameter $\Delta_{\text {Laser_Intensity }}$ is defined through laser line intensity analysis over the material on which the measurements will be taken.

In fig. 5 it is possible to visualize the result of the laser line detection by both cameras, after the laser line detection has carried out the calibration of horizontal and vertical images.

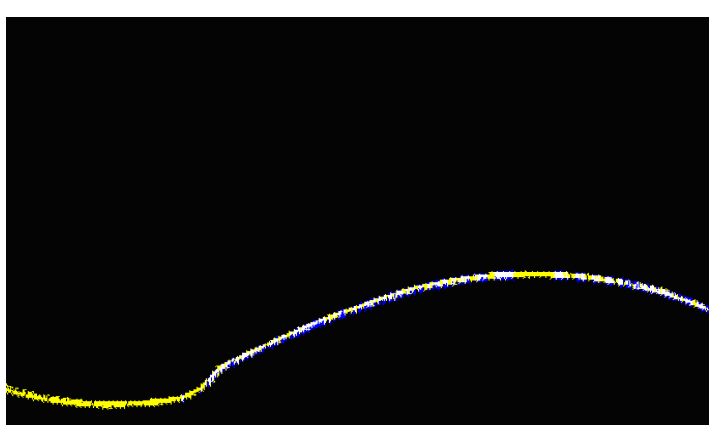

Fig. 5 - Result of the laser line detection in both cameras: yellow - camera 1 image, blue - camera 2 image, white camera 1 image and camera 2 image intersection.

The second step of the laser line detection consists of detecting the laser line centre, which corresponds to the average value between the maximum and the minimum values of the same. This step is carried out after step 2.B and before step 2.C of the calibration process. After laser line detection in both image cameras, step 2.A and 2.B of the calibration process are carried out. Then a logic operation is carried out between the two images resulting from calibration. In the resultant image of the logical operation, the laser line centre is detected (Fig. 6 ). This image is then ready for the execution of 2.C., the final step in calibration. 


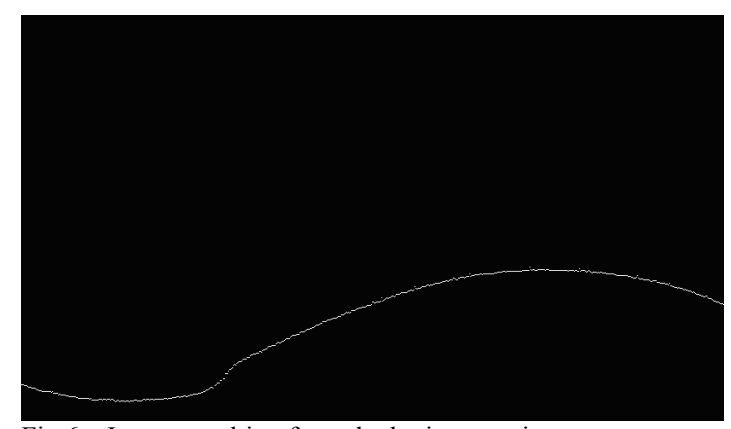

Fig.6 - Image resulting from the logic operation.

\section{RESULTS AND DISCUSSION}

A calibre (Fig. 7) was used for machine calibration process with the following characteristics: steps of approximately $10 \mathrm{~mm}$, the exact value of the height and length of each step was gotten using a CMM (Contact Measure Machine) of high precision (Mitutoyo, Class 0 , Precision $0.1 \mu \mathrm{m}$ ).

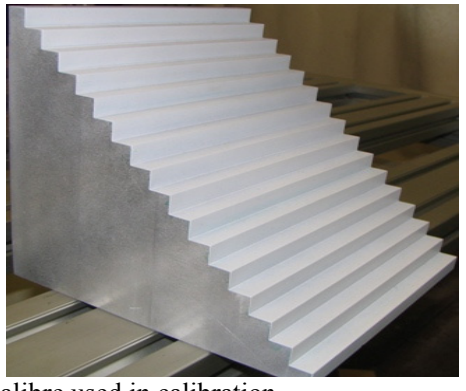

Fig. 7 - Calibre used in calibration.

Then a set of measurements on calibre steps (height and length) was carried out using the system developed. The measurements had been carried out with the calibre placed in different image points with special incidence in the image extremities because those are the points of greatest distortion for the calibration process. Fig.8 and Fig.9 show the resulting measurements made by the system under development; $\mathrm{X}$ axis (horizontal) and $\mathrm{Y}$ axis (vertical) respectively.

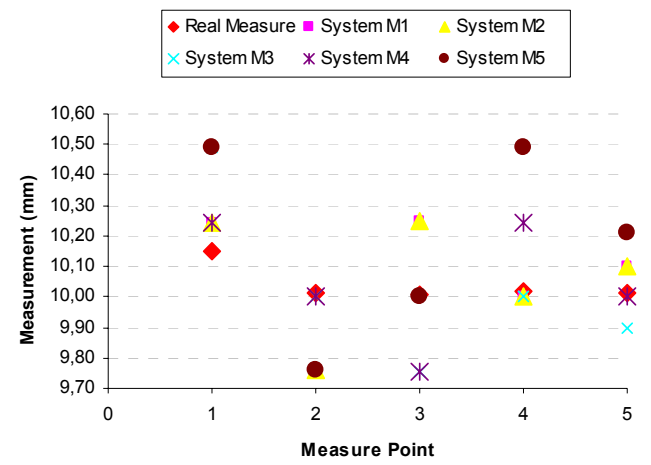

Fig. 8- Chart with five calibre vertical step measurements (X axis) on five distinguished image points (System M1 to M5).

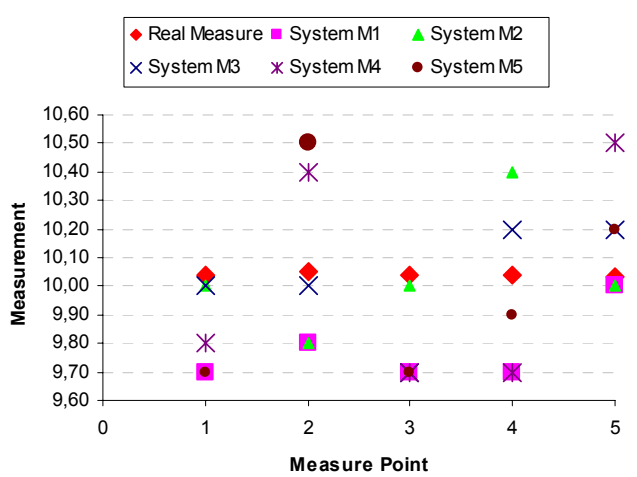

Fig. 9- Chart with five calibre vertical step measurements (X axis) on five determined image points (System M1 to M5).

TABLE I

CALIBRATION PROCEDURE STATISTICS

\begin{tabular}{|c|c|c|c|}
\cline { 2 - 4 } \multicolumn{1}{c|}{} & $\begin{array}{c}\text { Mean } \\
\text { Deviation }(\mathbf{m m})\end{array}$ & $\begin{array}{c}\text { Mean } \\
\text { Error }(\mathbf{m m})\end{array}$ & $\begin{array}{c}\text { Maximal } \\
\text { Error }(\mathbf{m m})\end{array}$ \\
\hline $\mathbf{X}$ & 0,165 & 0,199 & 0,465 \\
\hline $\mathbf{Y}$ & 0,147 & 0,177 & 0,470 \\
\hline
\end{tabular}

From the analysis of the results obained, it is possible to evidence that:

- The maximum error is inferior to 2 pixels, which expresses a system reading error inferior at $0,5 \mathrm{~mm}$. The system error subject to improvement by using higher resolution cameras or lenses that reduce the field of vision.

- The system presents a $0.25 \mathrm{~mm} /$ pixel horizontal resolution ( $\mathrm{X}$ axis), and a $0.33 \mathrm{~mm} /$ pixel vertical resolution, in a field of vision 170x160 mm.

- The mean deviation is inferior to the system resolution.

- The mean error is inferior to the system resolution.

- Ease of laser stripe detection in surfaces with high level of laser line absorption and complex geometry.

The results obtained validate the use of this calibration procedure in the system under development, a flexible quality control system for 3D object surfaces.

\section{CONCLUSION}

This paper presented a new calibration procedure for stereo vision and laser strip detection.

The new procedure has the following features:

- only uses laser-coplanar points;

- oriented towards laser line detection;

- permits the correspondence of two images on the laser plane without the use of a rigid motion equation. 
These features make this procedure simple and consequently easier to implement.

\section{FUTURE WORK}

The aim of this project is to develop a flexible quality control system for 3D object surfaces. The system developed has a total scanning area of $1000 \times 1000 \mathrm{~mm}$ and a field of vision of 170 x $160 \mathrm{~mm}$. It will be necessary to integrate multiple range images and develop a complete set of algorithms that will allow analysis of $3 \mathrm{D}$ surfaces for generating quality control reports.

\section{ACKNOWLEDGMENTS}

This work has being supported by "Fundação para a Ciência e a Tecnologia" (Portugal) through the $\mathrm{PhD}$ grant with the reference SFRH/BD/18155/2004.

\section{REFERENCES}

[1] Hong-Tzong Yau, Chun-Yan Chen, Robert G. Wilhelm, Registration and integration of multiple laser scanned data for reverse Engineering of complex 3D models, int. j. prod. res., 2000, vol. 38, no. 2, 269 \pm 285

[2] J.J. Aguilar, F. Torres, M. A. Lope, Stereo Vision for 3D measurement: accuracy analysis, calibration and industrial applications, Measurement Vol. 18, No. 4 pp. 193-200,1996.

[3] Barone, Sandro, Bruno, Stereo Vision and laser stripers for three-dimensional surface measurements, Andrea, XVI Congreso International de Ingeniería Gráfica, 2004

[4] F. Devernay and O. Faugeras. Computing differential properties of 3-D shapes from stereoscopic images without 3-D models. In CVPR'94, Seattle, USA, pages 208-213, 1994

[5] Reimar K. Lenz, Roger Y. Tsay, Techniques for Calibration of Scale Factor and Image Center for High Accuracy 3-D Machine Vision Metrology, IEEE Transactions on Pattern Analysis and Machine Intelligence, vol. 10, No. 5 September 1988.

[6] R.Y. Tsai, A Versatile camera calibration technique for high accuracy $3 \mathrm{D}$ machine vision metrology using off-the-shelf TV cameras and lenses, IBM Res. Rep. RC 51342, May 8, 1985;

[7] Heikkila. J., Silve' n O., 'A four-step camera calibration procedure with implicit image correction,', Proc. CVPR '97, IEEE, 1997, pp. 1106-1112.

[8] João G. França, Mário A. Gazziro, Alessandro N. Ide, José H. Saito, A 3D
Scanning System Based on laser Triangulation and Variable Field of View, Conference on Image Processing, IEEE, 2005. 